\title{
Predicting the progression of coronary artery calcification
}

Measuring the degree of coronary artery calcification (CAC) using CT enables physicians to quantify coronary artery disease progression. Investigators from Germany, led by Dr Raimund Erbel and Dr Nils Lehmann, conducted the Heinz Nixdorf Recall study to "prove that noninvasive imaging of the coronary arteries by CT and measurement of calcium load ... could improve coronary risk prediction".

CAC percentiles at baseline and after 5 years were measured in 3,481 participants. The most important finding from this study, described by Dr Erbel as "astonishing", is that the progression of CAC seems to be almost inevitable, with a very high explained variance, and minimal influence from cardiovascular risk factors. The investigators developed a mathematical tool to predict the degree of calcification for each individual, and identified only a very small mean difference between observed and predicted CAC progression. The tool can be used to calculate the degree of CAC progression on the basis of age, sex, and percentile of CAC distribution for any given time span. The finding "offers the opportunity to initiate re-scans ... [when] certain CAC thresholds can be expected, and to avoid unnecessary CT scans in between".

Given that the progression of CAC seems to follow a genetically determined, heritable pathway, Dr Erbel emphasizes the need to conduct the subsequent Heinz Nixdorf Recall Multi Generation study "to measure ... the CAC in the next generation of the study cohort ... to check the genetic background and the heritability, and study the influence of epigenetic factors".

\section{Karina Huynh}

Original article Erbel, R. et al. Progression of coronary artery calcification seems to be inevitable, but predictableresults of the Heinz Nixdorf Recall (HNR) study. Eur. Heart J. doi:10.1093/eurheartj/ehu288 